\title{
Усиление терагерцового излучения в плазмонной $n-i-p-i$-структуре на основе графена с инжекцией носителей заряда
}

\author{
(C) О.В. Полищук ${ }^{1}$, Д.В. Фатеев ${ }^{1}$, В.В. Попов ${ }^{1,2}$ \\ ${ }^{1}$ Саратовский филиал Института радиотехники и электроники им. В.А. Котельникова \\ Российской академии наук, \\ 410019 Саратов, Россия \\ ${ }^{2}$ Саратовский государственный университет, \\ 410012 Саратов, Россия \\ E-mail: polischuk.sfire@mail.ru, popov_slava@yahoo.co.uk \\ (Получане 27 апреля 2017 г. Принята к печати 12 мая 2017 г.)
}

\begin{abstract}
Теоретически исследован спектр поглощения/усиления терагерцового излучения в неоднородном графене $(n-i-p-i$-структура) с периодической двойной металлической решеткой. Показано, что усиление терагерцового излучения на частоте плазмонного резонанса резко возрастает, когда потери за счет электронного рассеяния и потери на излучение уравновешиваются плазмонным усилением (связанным со стимулированной излучательной межзонной рекомбинацией электронно-дырочных пар в инвертированной области графена).
\end{abstract}

DOI: $10.21883 /$ FTP.2017.11.45102.16

\section{1. Введение}

Графен - двумерный монослой графита с бесщелевым линейным (дираковским) энергетическим спектром носителей заряда [1] обладает сильным плазмонным откликом на терагерцовых (ТГц) частотах, что обусловлено как высокой плотностью, так и малой коллективной эффективной массой свободных носителей заряда [2,3]. Использование плазменных колебаний носителей заряда в графене представляется привлекательным, поскольку позволяет сконцентрировать электромагнитное поле вблизи графена и, таким образом, значительно повысить эффективность взаимодействия ТГц излучения с графеном. Это является важным как с точки зрения изучения физических свойств самого графена, так и для создания ТГц устройств на основе графена.

Возникновение отрицательной высокочастотной дифференциальной проводимости графена в ТГц частотном диапазоне за счет оптической [4], инжекционной [5] и диффузионной [6] накачки было исследовано в целях создания ТГц графенового лазера. Наличие отрицательной дифференциальной проводимости может приводить к стимулированной генерации ТГц плазмонов в графене [7]. Вынужденное излучение фотонов в инвертированном графене недавно наблюдалось в ТГц диапазоне [8]. По сравнению с вынужденным излучением электромагнитных мод (фотонов), вынужденное излучение плазмонов при межзонных переходах носителей заряда в инвертированном графене является гораздо более сильным эффектом за счет малой групповой скорости плазмонов в графене и сильной локализации плазмонного поля вблизи графенового слоя [4].

В данной работе теоретически предсказана возможность гигантского усиления ТГц излучения в неоднородном графене с инжекцией носителей заряда $(n-i-p-i$-структура) с латеральной периодической двойной металлической решеткой. Инжекционная накач- ка более проста в технологическом исполнении и энергетически более выгодна по сравнению с оптической накачкой.

\section{2. Постановка задачи и метод решения}

Рассмотрим бесконечный лист периодически структурированного графена, расположенный в плоскости $y=0$ на поверхности подложки с вещественной диэлектрической постоянной $\varepsilon_{s}$ (один период структуры показан на рис. 1). Планарная двойная периодическая решетка представляется последовательностью проводящих металлических полосок нулевой толщины с периодом $L$, расположенных в плоскости $y=d$ и отделенных от графена слоем диэлектрика с вещественной диэлектрической постоянной $\varepsilon_{b}$. Каждая проводящая полоска решетки шириной $w$ бесконечна в z-направлении и отделена от соседних полосок разной ширины щелями. Проводимость двумерных металлических полосок периодической решетки (Au в нашем случае) полагалась равной $\sigma_{\mathrm{Me}}=2.5 \mathrm{OM}^{-1}$.

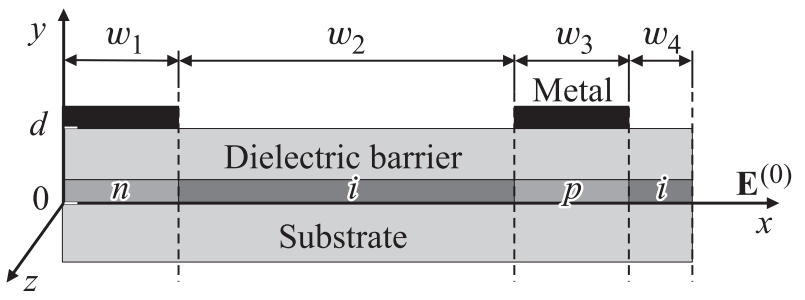

Рис. 1. Схематическое изображение одного периода исследуемой структуры и система координат. Внешняя ТГц волна падает сверху в направлении нормали к плоскости структуры с поляризацией электрического поля поперек полосок металлической решетки. 
Окружающая среда при $y>d$ имеет диэлектрическую постоянную $\varepsilon_{a}=1$ (вакуум). Подача разнополярного электрического потенциала на соседние электроды металлической решетки приводит к созданию $n$ - и $p$-областей в графене (рис. 1), для которых проводимость электрически допированного графена определяется формулой $[9,10]$ :

$$
\begin{aligned}
& \sigma_{\mathrm{Gr}}^{n, p}(\omega)=\frac{e^{2} 8 \tau k_{\mathrm{B}} T}{4 \pi \hbar^{2}(1-i \omega \tau)} \ln \left[2 \cosh \left(\frac{\mathscr{E}_{\mathrm{F}}}{2 k_{\mathrm{B} T}}\right)\right] \\
&+ \frac{e^{2}}{4 \hbar} G\left(\frac{\hbar \omega}{2}\right)-\frac{e^{2} \omega}{i \pi} \int_{0}^{\infty} \frac{G(\mathscr{E})-G(\hbar \omega / 2)}{(\hbar \omega)^{2}-4 \mathscr{E}^{2}} d \mathscr{E}, \\
& G(\mathscr{E})=\frac{\sinh \left(\mathscr{E} / k_{\mathrm{B}} T\right)}{\cosh \left(\mathscr{E} / k_{\mathrm{B}} T\right)+\cosh \left(\mathscr{E}_{\mathrm{F}} / k_{\mathrm{B}} T\right)} .
\end{aligned}
$$

Здесь $\mathscr{E}_{\mathrm{F}}-$ энергия Ферми, температура $T$ полагается равной $300 \mathrm{~K}, e-$ заряд электрона, $k_{\mathrm{B}}-$ постоянная Больцмана и $\hbar-$ приведенная постоянная Планка. Первое слагаемое в формуле (1) описывает отклик в форме Друде с участием внутризонных процессов, описываемых феноменологическим временем рассеяния носителей заряда в графене $\tau$. Второе слагаемое описывает межзонные переходы в инвертировнном графене. На рис. 2, а представлена действительная часть проводимости электрически допированного графена в зависимости от частоты падающего ТГц излучения и величины уровня Ферми.

Инжекционная накачка электронов из $n$-области и дырок из $p$-области позволяет создать инвертированное распределение носителей заряда в $i$-области графена, расположенной под широким просветом металлической решетки. В этом случае отклик графена в инвертированной $i$-области характеризуется комплексной динамической ТГц проводимостью $[4,5]$ :

$$
\begin{aligned}
\sigma_{\mathrm{Gr}}^{(i)}(\omega)= & \frac{e^{2} 8 k_{\mathrm{B}} T \tau}{4 \pi \hbar^{2}(1-i \omega \tau)} \ln \left[1+\exp \left(\frac{\mathscr{E}_{\mathrm{F}}}{k_{\mathrm{B}} T}\right)\right] \\
& +\frac{e^{2}}{4 \hbar} \tanh \left(\frac{\hbar \omega-2 \mathscr{E}_{F}}{4 k_{\mathrm{B}} T}\right) \\
& -\frac{e^{2} \omega}{i \pi} \int_{0}^{\infty} \frac{G\left(\mathscr{E}, \mathscr{E}_{\mathrm{F}}\right)-G\left(\hbar \omega / 2, \mathscr{E}_{\mathrm{F}}\right)}{(\hbar \omega)^{2}-4 \mathscr{E}^{2}} d \mathscr{E}, \\
G\left(\mathscr{E}, \mathscr{E}^{\prime}\right) & =\frac{\sinh \left(\mathscr{E} / k_{\mathrm{B}} T\right)}{\cosh \left(\mathscr{E} / k_{\mathrm{B}} T\right)+\cosh \left(\mathscr{E}^{\prime} / k_{\mathrm{B}} T\right)} .
\end{aligned}
$$

Квазиэнергия Ферми в широкой $i$-области графена определяется падением инжекционного напряжения на длине этой области $\mathscr{E}_{\mathrm{F}}=U_{\text {in }} / 2$. Инжекция носителей заряда в узкие $i$-области графена не происходит из-за обратного смещения $p-i-n$-структуры в этой области, таким образом, квазиэнергия Ферми равна нулю в $i$-области графена. Действительная часть проводимости (2), ответственная за диссипацию энергии и учитывающая
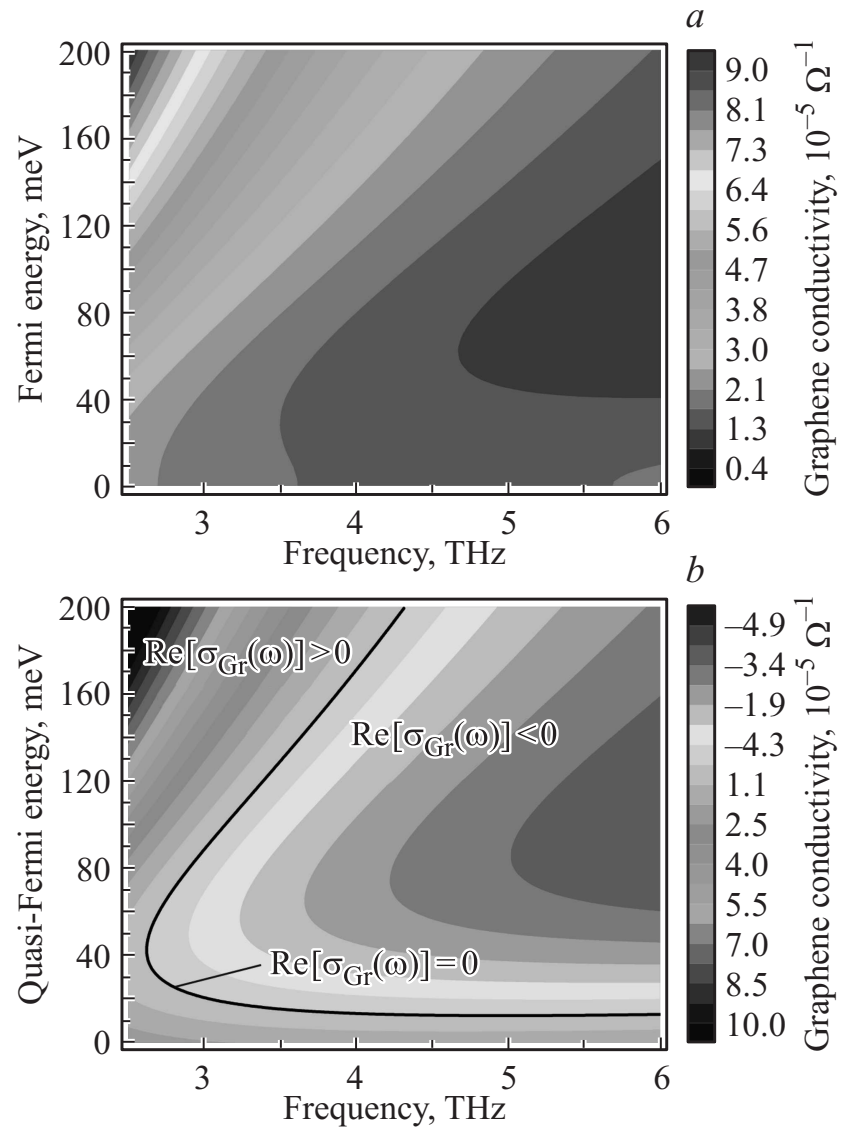

Рис. 2. Контурная карта действительной части проводимости электрически допированного графена $(a)$ и активного графена с инжекцией носителей заряда $(b)$ в зависимости от частоты и величины энергии (квазиэнергии) Ферми. Расчеты приведены для комнатной температуры и $\tau=10^{-12} \mathrm{c}$.

оба механизма рассеяния, представлена на рис. $2, \quad b$. Действительная часть проводимости инвертированной области графена $\sigma_{\mathrm{Gr}}^{(i)}(\omega)$ может принимать отрицательные значения при превышении пороговой величины накачки. Для осуществления инжекции носителей заряда в $i$-область необходимо, чтобы энергия Ферми в $p$ и $n$-областях графена была больше, чем квазиэнергия Ферми в $i$-области графена.

Будем считать, что плоская электромагнитная волна падает из вакуума нормально на плоскость структуры. Электрическое поле падающей волны $\mathbf{E}^{(0)} \exp \left[i k^{(0)} y-i \omega t\right]$, где $\omega-$ круговая частота, $k_{y}^{(0)}=$ $=k_{0}=\omega / c, c-$ скорость света в вакууме, поляризовано поперек полосок решетки (вдоль оси $x$ ).

Задача решалась с использованием самосогласованного электродинамического подхода. В рассматриваемой геометрии индуцированное электрическое поле имеет ненулевые $x$ - и $y$-компоненты, тогда как индуцированное магнитное поле имеет только ненулевую $z$-компоненту ( $p$-поляризация). В силу периодичности структуры в $x$-направлении индуцированные магнитное и электриче- 
ское поля можно представить в виде ряда Фурье:

$$
\begin{gathered}
H_{z}(x, y)=\sum_{m=-\infty}^{\infty} H_{z m}(y) \exp \left(i q_{m} x\right), \\
E_{x z}(x, y)=\sum_{m=-\infty}^{\infty} E_{x m, z m}(y) \exp \left(i q_{m} x\right)
\end{gathered}
$$

где

$$
\begin{gathered}
H_{z m}(y)=\frac{1}{L} \int_{-L / 2}^{L / 2} H_{z}(x, y) \exp \left(-i q_{m} x\right), \\
E_{x m, z m}(y)=\frac{1}{L} \int_{-L / 2}^{L / 2} E_{x, z}(x, y) \exp \left(-i q_{m} x\right)
\end{gathered}
$$

- амплитуды $m$-й фурье-гармоники соответственно магнитного и электрического полей и $q_{m}=2 \pi m / L$ $(m= \pm 1, \pm 2, \pm 3 \ldots)$.

Используя уравнения (3) для каждой искомой компоненты поля, запишем систему уравнений Максвелла в каждой среде в фурье-представлении. В этом представлении, граничные условия для тангенциальных составляющих электрического поля $E_{x}$ и магнитного поля $H_{z}$ записываются в виде

$$
\begin{gathered}
E_{x m}^{(b)}(0)=E_{x m}^{(s)}(0), \\
H_{z m}^{(b)}(0)-H_{z m}^{(s)}(0)=-I_{x m}(0)
\end{gathered}
$$

в плоскости $y=0$ и

$$
\begin{gathered}
E_{x m}^{(a)}(d)+E^{(0)} \exp \left(i k_{0} d\right)=E_{x m}^{(b)}(d), \\
H_{z m}^{(a)}(d)+H^{(0)} \exp \left(i k_{0} d\right)-H_{z m}^{(b)}(d)=-I_{x m}(d),
\end{gathered}
$$

в плоскости $y=d$, где $I_{x m}(0)$ и $I_{x m}(d)$ представляют собой амплитуды $m$-ой фурье-гармоники поверхностной плотности тока соответственно в графене и в плоскости металлических полосок решетки. Индексы $a, b$ и $s$ относятся соответственно к окружающей среде (вакуум в рассматриваемом случае), барьерному слою и подложке. Амплитуды фурье-гармоник индуцированного магнитного поля в различных средах записываются в виде

$$
\begin{aligned}
H_{z m}^{(a)}(y)= & A_{m} \exp \left(i \alpha_{m}^{(a)} y\right), & & y \geq d, \\
H_{z m}^{(b)}(y)= & B_{m} \exp \left(i \alpha_{m}^{(b)} y\right) & & \\
& +C_{m} \exp \left(-i \alpha_{m}^{(b)} y\right), & & 0 \leq y \leq d, \\
H_{z m}^{(s)}(y)= & D_{m} \exp \left(i \alpha_{m}^{(s)} y\right), & & y \leq 0,
\end{aligned}
$$

где $\alpha_{m}^{(j)}= \pm \sqrt{k_{0}^{2} \varepsilon_{j}-q_{m}^{2}}(j=a, b, s)$ - поперечное волновое число $m$-ой пространственной гармоники в $j$-й среде. Используя уравнения (5-7) и уравнения Максвелла в каждой среде в фурье-представлении, после очевидных алгебраических преобразований получим следующие соотношения между тангенциальными фурье-компонентами электрического поля и поверхност- ной плотности тока в плоскостях графенового слоя и металлической решетки:

$$
\begin{gathered}
E_{x m}^{(b)}(d)=Z_{m}^{1,1} j_{x m}(d)+Z_{m}^{(1,2)} j_{x m}(0)+\delta_{m 0} Z^{(1)} E_{x}^{(0)}, \\
E_{x m}^{(b)}(0)=Z_{m}^{2,1} j_{x m}(d)+Z_{m}^{(2,2)} j_{x m}(0)+\delta_{m 0} Z^{(2)} E_{x}^{(0)},
\end{gathered}
$$

где

$$
\begin{gathered}
Z_{m}^{(1,1)}=-\frac{\alpha_{m}^{(a)} \alpha_{m}^{(b)} \alpha_{m}^{(s)} \varepsilon_{b}}{\omega \varepsilon_{0} \chi_{m}}\left(1+\exp \left(2 i \alpha_{m}^{(b)} d\right)\right) \\
+\frac{\alpha_{m}^{(a)} \alpha_{m}^{(b)} \alpha_{m}^{(s)} \varepsilon_{s}}{\omega \varepsilon_{0} \chi_{m}}\left(1-\exp \left(2 i \alpha_{m}^{(b)} d\right)\right) \\
Z_{m}^{1,2}=-\frac{\alpha_{m}^{(a)} \alpha_{m}^{(b)} \alpha_{m}^{(s)} \varepsilon_{b}}{\omega \varepsilon_{0} \chi_{m}} 2 \exp \left(i \alpha_{m}^{(b)} d\right) \\
Z_{m}^{2,1}=-\frac{\alpha_{m}^{(a)} \alpha_{m}^{(b)} \alpha_{m}^{(s)} \varepsilon_{b}}{\omega \varepsilon_{0} \chi_{m}} 2 \exp \left(i \alpha_{m}^{(b)} d\right)=Z_{m}^{1,2} \\
Z_{m}^{(2,2)}=-\frac{\alpha_{m}^{(a)} \alpha_{m}^{(b)} \alpha_{m}^{(s)} \varepsilon_{a}}{\omega_{0} \varepsilon \chi_{m}}\left(1-\exp \left(2 i \alpha_{m}^{(b)} d\right)\right) \\
-\frac{\alpha_{m}^{(a)} \alpha_{m}^{(b)} \alpha_{m}^{(s)} \varepsilon_{b}}{\omega \varepsilon_{0} \chi_{m}}\left(1+\exp \left(-2 i \alpha_{m}^{(b)} d\right)\right) \\
Z^{(1)}=\frac{\varepsilon_{0} \varepsilon_{a} \omega}{\alpha_{m}^{(a)}} \delta_{m 0} 2 \exp \left(-i \alpha_{m}^{(a)} d\right) Z_{m=0}^{(1,1)}, \\
Z^{(2)}=\frac{\varepsilon_{0} \varepsilon_{a} \omega}{\alpha_{m}^{(a)}} \delta_{m 0} 2 \exp \left(-i \alpha_{m}^{(a)} d\right) Z_{m=0}^{(2,1)} \\
\chi_{m}=\left(\alpha_{m}^{(b)} \varepsilon_{a}+\alpha_{m}^{(a)} \varepsilon_{b}\right)\left(\alpha_{m}^{(s)} \varepsilon_{b}-\alpha_{m}^{(b)} \varepsilon_{s}\right) \\
\exp \left(2 i \alpha_{m}^{(b)} d\right)\left(\alpha_{m}^{(b)} \varepsilon_{a}-\alpha_{m}^{(a)} \varepsilon_{b}\right)\left(\alpha_{m}^{(s)} \varepsilon_{b}+\alpha_{m}^{(b)} \varepsilon_{s}\right)
\end{gathered}
$$

Запишем локальный закон Ома $j(\omega, x)=$ $=\sigma(\omega, x) E(\omega, x)$, принимая во внимание явный вид разрывной функции проводимости $\sigma(\omega, x)$ от координат:

$$
\sigma(\omega, x)=\left\{\begin{array}{lll}
\sigma_{w 1,0} & \text { при } & 0<x<w_{1}, \\
\sigma_{w 2,0} & \text { при } & w_{1}<x<w_{2}, \\
\sigma_{w 3,0} & \text { при } & w_{2}<x<w_{3}, \\
\sigma_{w 4,0} & \text { при } & w_{3}<x<L
\end{array}\right.
$$

в плоскости $y=0$ и

$$
\sigma(\omega, x)=\left\{\begin{array}{lll}
\sigma_{w 1, d} & \text { при } & 0<x<w_{1}, \\
\sigma_{w 2, d} & \text { при } & w_{1}<x<w_{2}, \\
\sigma_{w 3, d} & \text { при } & w_{2}<x<w_{3}, \\
\sigma_{w 4, d} & \text { при } & w_{3}<x<L
\end{array}\right.
$$

в плоскости $y=d$. Для данной геометрии структуры (см. рис. 1) $\sigma_{w 1,0}=\sigma_{w 3,0}=\sigma_{\mathrm{Gr}}^{(n, p)}(\omega), \sigma_{w 2,0}=\sigma_{\mathrm{Gr}}^{(i)}(\omega)$, $\sigma_{w 4,0}=\sigma_{\mathrm{Gr}}^{(i)}(\omega), \quad$ при $\quad \mathscr{E}_{\mathrm{F}}=0, \quad \sigma_{w 1, d}=\sigma_{w 3, d}=\sigma_{\mathrm{Me}}$, $\sigma_{w 2, d}=\sigma_{w 4, d}=0$. Сформулируем систему, состоящую из восьми интегральных уравнений относительно поверхностной плотности тока $j_{x}^{w_{k}}(x, y)$ на четырех различных участках периода $(k=1,2,3,4), \quad$ в 
плоскости металлической решетки $y=d$ :

$$
\begin{aligned}
j_{x}^{w_{k}}(x, d) & -\sigma_{w_{k}, d} Z^{(1)} F_{x}^{(0)} \delta_{m 0}=\int_{0}^{w_{1}} j_{x}^{w_{1}}\left(x^{\prime}, d\right) G_{m, k}^{1,1}\left(x, x^{\prime}, d\right) d x^{\prime} \\
& +\int_{w_{1}}^{w_{2}} j_{x}^{w_{2}}\left(x^{\prime}, d\right) G_{m, k}^{1,1}\left(x, x^{\prime}, d\right) d x^{\prime} \\
& +\int_{w_{2}}^{w_{3}} j_{x}^{w_{3}}\left(x^{\prime}, d\right) G_{m, k}^{1,1}\left(x, x^{\prime}, d\right) d x^{\prime} \\
& +\int_{w_{3}}^{L} j_{x, \omega_{0}}^{w_{4}}\left(x^{\prime}, d\right) G_{m, k}^{1,1}\left(x, x^{\prime}, d\right) d x^{\prime} \\
& +\int_{0}^{w_{1}} j_{x}^{w_{1}}\left(x^{\prime}, 0\right) G_{m, k}^{1,2}\left(x, x^{\prime}, d\right) d x^{\prime} \\
& +\int_{w_{1}}^{w_{2}} j_{x}^{w_{2}}\left(x^{\prime}, 0\right) G_{m, k}^{1,2}\left(x, x^{\prime}, d\right) d x^{\prime} \\
& +\int_{w_{2}}^{w_{3}} j_{x}^{w_{3}}\left(x^{\prime}, 0\right) G_{m, k}^{1,2}\left(x, x^{\prime}, d\right) d x^{\prime} \\
& +\int_{w_{3}}^{L} j_{x, \omega_{0}}^{w_{4}}\left(x^{\prime}, 0\right) G_{m, k}^{1,2}\left(x, x^{\prime}, d\right) d x^{\prime}
\end{aligned}
$$

и в плоскости графенового слоя $y=0$ :

$$
\begin{aligned}
j_{x}^{\left(w_{k}\right)}(x, 0) & -\sigma_{w_{k}, 0} Z^{(2)} E_{x}^{(0)} \delta_{m 0} \\
= & \int_{0}^{w_{1}} j_{x}^{(w 1)}\left(x^{\prime}, d\right) G_{m, k}^{(2,1)}\left(x, x^{\prime}, 0\right) d x^{\prime} \\
& +\int_{w_{1}}^{w_{2}} j_{x}^{(w 2)}\left(x^{\prime}, d\right) G_{m, k}^{(2,1)}\left(x, x^{\prime}, 0\right) d x^{\prime} \\
& +\int_{w_{2}}^{w_{3}} j_{x}^{(w 3)}\left(x^{\prime}, d\right) G_{m, k}^{(2,1)}\left(x, x^{\prime}, 0\right) d x^{\prime} \\
& +\int_{w_{3}}^{L} j_{x}^{(w 4)}\left(x^{\prime}, d\right) G_{m, k}^{(2,1)}\left(x, x^{\prime}, 0\right) d x^{\prime} \\
& +\int_{0}^{w_{1}} j_{x}^{(w 1)}\left(x^{\prime}, 0\right) G_{m, k}^{(2,2)}\left(x, x^{\prime}, 0\right) d x^{\prime} \\
& +\int_{w_{2}}^{w_{2}} j_{x}^{(w 2)}\left(x^{\prime}, 0\right) G_{m, k}^{(2,2)}\left(x, x^{\prime}, 0\right) d x^{\prime} \\
& +\int_{w_{2}}^{w_{3}} j_{x}^{(w 3)}\left(x^{\prime}, 0\right) G_{m, k}^{(2,2)}\left(x, x^{\prime}, 0\right) d x^{\prime} \\
& +\int_{w_{3}}^{L} j_{x}^{(w 4)}\left(x^{\prime}, 0\right) G_{m, k}^{(2,2)}\left(x, x^{\prime}, 0\right) d x^{\prime}
\end{aligned}
$$

где

$$
\begin{aligned}
& G_{m, k}^{(1,1)}\left(x, x^{\prime}\right)=\sigma_{w_{k}, d} \frac{1}{L} \sum_{m} Z_{m}^{1,1} \exp \left(i q_{m}\left(x-x^{\prime}\right)\right), \\
& G_{m, k}^{(1,2)}\left(x, x^{\prime}\right)=\sigma_{w_{k}, d} \frac{1}{L} \sum_{m} Z_{m}^{1,2} \exp \left(i q_{m}\left(x-x^{\prime}\right)\right), \\
& G_{m, k}^{(2,1)}\left(x, x^{\prime}\right)=\sigma_{w_{k}, 0} \frac{1}{L} \sum_{m} Z_{m}^{1,2} \exp \left(i q_{m}\left(x-x^{\prime}\right)\right), \\
& G_{m, k}^{(2,2)}\left(x, x^{\prime}\right)=\sigma_{w_{k}, 0} \frac{1}{L} \sum_{m} Z_{m}^{2,2} \exp \left(i q_{m}\left(x-x^{\prime}\right)\right)
\end{aligned}
$$

- ядра интегральных уравнений.

Полученная система интегральных уравнений Фредгольма второго рода (10)-(11) решалась численно методом Галеркина с разложением восьми искомых функций $(x$-компонент плотности поверхностного электрического тока $j_{x}^{\left(w_{k}\right)}(x, y)$ в ряд на каждом участке периода) по ортогональным полиномам Лежандра. Индуцированные электрические и магнитные поля в любой точке структуры могут быть найдены с помощью выражений (8) и уравнений Максвелла в каждой из сред.

Длина волны резонансной плазмонной моды, возбуждаемой в графене падающей ТГц волной, определяется периодом $L$ металлической решетки. Поскольку длина волны плазмона в графене гораздо (на 2-3 порядка) короче, чем длина электромагнитной волны на той же частоте $[2,3]$, для возбуждения плазмонных резонансов в структуре необходимо выполнение условия $L \ll 2 \pi / k_{0}$. В этом случае только нулевые фурье-гармоники $(m=0)$ соответствуют бегущим волнам, излучаемым в окружающую среду и в подложку, а все фурье-гармоники более высокого порядка характеризуют эванесцентные поля, убывающие при $y \rightarrow \pm \infty$. Следовательно, коэффициенты отражения $R$ и пропускания $T$ исследуемой структуры в области дальнего поля могут быть вычислены соответственно как $R=\left|E_{x 0}(d)^{2}\right| /\left|E^{(0)}\right|^{2}$ и $T=\left|E_{x 0}(d)\right|^{2} \sqrt{\varepsilon_{s}} /\left|E^{(0)}\right|^{2}$. Коэффициент поглощения/усиления рассчитывается как $A=P_{\text {abs }} / P_{0}$, где $P_{0}-$ ТГц мощность, падающая на период структуры (на единицу ширины структуры);

$$
P_{\text {abs }}=\frac{1}{2 L} \int_{0}^{L} \operatorname{Re}[\sigma(\omega, x)]\left|E_{x}(x, 0)\right|^{2} d x
$$

- поглощенная ТГц мощность на периоде структуры (на единицу ширины структуры). Закон сохранения энергии $R+T+A=1$ выполняется как в режиме поглощения $(A>0)$, так и в режиме усиления $(A<0)$.

Расчеты выполнены для реалистичных параметров исследуемой структуры (рис. 1) с периодом 1.2 мкм при комнатной температуре. Период структуры состоит из четырех участков: $w_{1}=w_{3}=0.3, \quad w_{2}=0.5$, $w_{4}=0.1$ мкм. Толщина барьерного слоя $d$ составляет 80 нм, диэлектрическая постоянная барьерного слоя и диэлектрической подложки $(\mathrm{Si})-11.7$. Энергия Ферми электронов в $n$-области и дырок в $p$-области электрически допированного графена полагалась равной 200 мэВ. 


\section{3. Результаты расчетов и их обсуждение}

Планарная металлическая периодическая решетка представляет собой в данной структуре эффективную антенну для падающего ТГц излучения [11]. С другой стороны, она образует распределенный планарный резонатор для плазмонных мод, возбуждаемых или генерируемых в графене соответственно падающим ТГц излучением или стимулированной плазмонной эмиссией.

Графен с двойной металлической периодической решеткой поддерживает плазмонные коллективные моды, когерентно колеблющиеся на всей площади структуры, что приводит к ТГц сверхизлучению из структуры на частотах плазмонного резонанса [12,13]. Светлый лепесток (рис. 3, $a$ ) соответствует плазмонному резонансу в режиме усиления. Усиление на частоте плазмонного резонанса резко возрастает (при соответствующем рез-
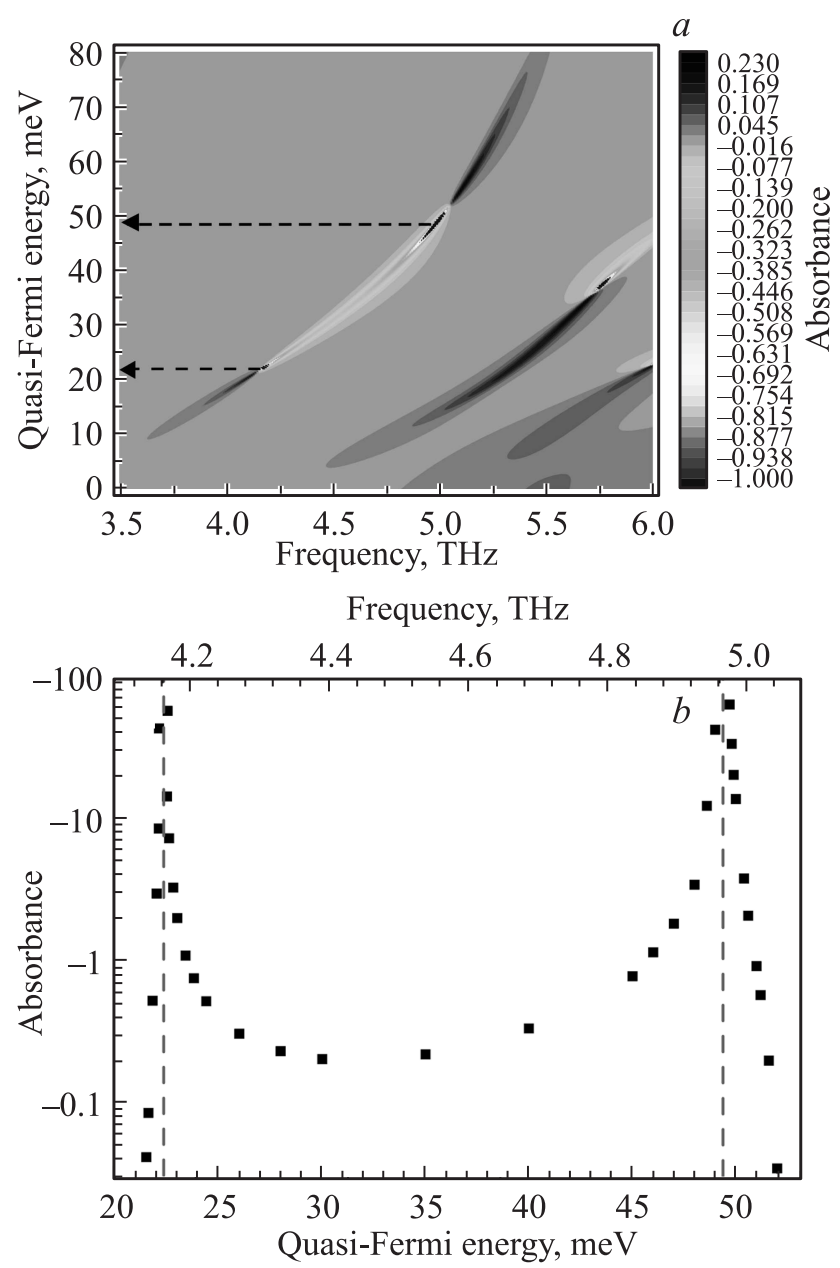

Pис. 3. Коэффициент поглощения/усиления ТГц мощности в зависимости от квазиэнергии Ферми и частоты падающей ТГц волны для плазменных резонансов $(a)$. Изменение коэффициента усиления вдоль лепестка плазмонного резонанса $(b)$. Период металлической решетки $L=1.2$ мкм, ширина области активного графена 0.5 мкм, электрически допированного графена -0.3 мкм. Толщина барьерного слоя $(\mathrm{Si}) d=80$ нм. ком уменьшении ширины линии усиления) при определенном значении квазиэнергии Ферми. Это соответствует режиму самовозбуждения коллективных плазмонных мод в графене с двойной металлической периодической решеткой и, как следствие, ТГц генерации из структуры. Генерация наступает, когда суммарные потери энергии за счет электронного рассеяния в графене и излучения электромагнитной энергии из структуры компенсируются плазмонным усилением, что обеспечивает высокую когерентность коллективной плазмонной моды. Значения квазиэнергии Ферми, соответствующие ТГц генерации на основном плазмонном резонансе, отмечены стрелками на рис. 3,a. Изменение коэффициента усиления вдоль лепестка основного плазмонного резонанса в окрестности режима самовозбуждения показано на рис. $3, b$. Видно, что условие стимулированной генерации ТГц излучения может быть реализовано на двух различных частотах (показанных штриховыми линиями на рис. 3,b) при различных значениях квазиэнергии Ферми. Такое поведение связано со сверхизлучательным характером коллективной плазмонной моды. В режиме плазмонного сверхизлучения радиационное затухание растет пропорционально концентрации носителей заряда, колеблющихся в коллективной плазмонной моде [14], тогда как диссипативное затухание плазмонов равно $1 / 2 \tau[15]$ и не зависит от концентрации носителей заряда в графене. Поскольку концентрация носителей заряда в графене связана с энергией Ферми как $N_{n(p)} \propto \mathscr{E}_{\mathrm{F}}^{2} /\left(\pi \hbar^{2} V_{\mathrm{F}}^{2}\right)$, где $V_{\mathrm{F}}=10^{8} \mathrm{~cm} / \mathrm{c}-$ скорость Ферми, то радиационное затухание растет пропорционально квадрату энергии Ферми. В то же время плазмонное усиление в заданном частотном интервале насыщается с ростом квазиэнергии Ферми вследствие конечной плотности состояний энергии носителей заряда в графене. В результате кривые зависимостей суммарного (радиационного плюс диссипативного) затухания и плазмонного усиления от мощности накачки пересекаются в двух точках [16], соответствующих разным режимам генерации.

\section{4. Заключение}

Периодическая $n-i-p-i$-структура на основе графена с инжекцией носителей заряда поддерживает плазмонные коллективные моды, когерентно колеблющиеся на всей площади структуры. Теоретические расчеты показывают, что суммарные потери энергии за счет электронного рассеяния в графене и излучения электромагнитной энергии из структуры могут быть скомпенсированы плазмонным усилением в области инвертированного графена. Это обеспечивает высокую когерентность коллективной плазмонной моды и приводит к ТГц сверхизлучению из структуры на частотах плазмонного резонанса. Резонансная частота плазменных колебаний в графене может варьироваться как положением квазиуровня Ферми, так и геометрией структуры. Полученные результаты показывают возможность создания 
компактных, частотно перестраиваемых наноразмерных плазмонных усилителей и генераторов в ТГц частотном диапазоне на основе графена с инжекционной накачкой.

Работа выполнена при поддержке гранта РФФИ № 16-02-00814.

\section{Список литературы}

[1] K.S. Novoselov, A.K. Geim, S.V. Morozov, D. Jiang, M.I. Katsnelson, I.V. Grigorieva, S.V. Dubonos, A.A. Firsov. Nature, 438, 197 (2005).

[2] J. Chen, M. Badioli, P. Alonso-González, S. Thongrattanasiri, F. Huth, J. Osmond, M. Spasenovic, A. Centeno, A. Pesquera, P. Godiquon, A.Z. Elorza, N. Camara, F.J. Garcia de Abajo, R. Hillenbrand, F.H. Koppens. Nature, 487, 77 (2012).

[3] Z. Fei, A.S. Rodin, G.O. Andreev, W. Bao, A.S. McLeod, M. Wagner, L.M. Zhang, Z. Zhao, M. Thiemens, G. Dominguez, M.M. Fogler, A.H. Casto Neto, C.N. Law, F. Keilmann, D.N. Basov. Nature, 487, 82 (2012).

[4] A. Dubinov, V.Y. Aleshkin, V. Mitin, T. Otsuji, V. Ryzhii. J. Phys.: Condens. Matter, 23, 145302 (2011).

[5] V. Ryzhii, M. Ryzhii, V. Mitin, T. Otsuji. J. Appl. Phys., 110, 094503 (2011).

[6] A.R. Davoyan, M.Yu. Morozov, V.V. Popov, A. Satou, T. Otsuji. Appl. Phys. Lett., 103, 251102 (2013).

[7] F. Rana. IEEE Trans. Nanotechn., 7, 91 (2008).

[8] S. Boubanga-Tombet, S. Chan, T. Watanabe, A. Satou, V. Ryzhii, T. Otsuji. Phys. Rev. B, 85, 035443 (2012).

[9] L.A. Falkovsky, A.A. Varlamov. Eur. Phys. J. B, 56, 281 (2007).

[10] M.S. Jang, V.W. Brar, M.C. Sherrott, J.J. Lopez, L. Kim, S. Kim, M. Choi, H.A. Atwater. Phys. Rev. B, 90, 165409 (2014).

[11] О.В. Полищук, В.В. Попов, В. Кнап. ФТП, 49 (1), 107 (2015).

[12] V.V. Popov, O.V. Polischuk, A.R. Davoyan, V. Ryzhii, T. Otsuji, M.S. Shur. Phys. Rev. B, 86, 195437 (2012).

[13] V.V. Popov, O.V. Polischuk, S.A. Nikitov, V. Ryzhii, T. Otsuji, M.S. Shur. J. Optics, 15, 114009 (2013).

[14] V.V. Popov, O.V. Polischuk, T.V. Teperik, X.G. Peralta, S.J. Allen, N.J.M. Horing, M.C. Wanke. J. Appl. Phys., 94, 3556 (2003).

[15] V.V. Popov, T.Yu. Bagaeva, T. Otsuji, V. Ryzhii. Phys. Rev. B, 81, 073404 (2010).

[16] О.В. Полищук, В.В. Попов, Т. Otsuji. ФТП, 49 (11), 1516 (2015).

Редактор Г.А. Оганесян

\section{Amplification of terahertz radiation by plasmons in the $n-i-p-i$-graphene structure with charge carrier injection}

\author{
O.V. Polischuk ${ }^{1}$, D.V. Fateev ${ }^{1}$, V.V. Popov ${ }^{\mathbf{1 , 2}}$ \\ ${ }^{1}$ Kotelnikov Institute of Radio Engineering and \\ Electronics, Russian Academy of Sciences, \\ 410019 Saratov, Russia \\ 2 National Research Saratov State University, \\ 410012 Saratov, Russia
}

\begin{abstract}
The absorption/amplification spectrum of terahertz radiation by plasmons in inhomogeneous graphene $(n-i-p-i-$ structure) with a planar dual metal grating is theoretically studied. It has been shown that the amplification of terahertz waves at the plasmon resonance frequencies dramatically increases when the total losses due to radiation and electron scattering are balanced by the plasmon gain (associated with the stimulated interband radiative recombination of electron-hole pairs in the region of the inverted graphene).
\end{abstract}

